\title{
Different milk feeding intensities during the first 4 weeks of rearing dairy calves: Part 3: Plasma metabolomics analysis reveals long-term metabolic imprinting in Holstein heifers
}

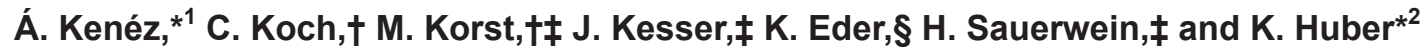 \\ *Institute of Animal Science, University of Hohenheim, Stuttgart 70599, Germany \\ †Educational and Research Centre for Animal Husbandry, Hofgut Neumühle, Münchweiler an der Alsenz 67729, Germany \\ ‡lnstitute for Animal Science, University of Bonn, Bonn 53115, Germany \\ §Institute of Animal Nutrition and Nutritional Physiology, Justus-Liebig University, Gießen 35392, Germany
}

\begin{abstract}
Adequate nutrition of calves is a fundamental requirement for efficient production in later life. Suboptimal nutrition before weaning could have detrimental longterm effects on metabolic health and could thereby decrease production efficiency. In this study, the metabolomic profiles of German Holstein calves reared on whole milk ad libitum $(\mathrm{n}=10)$, milk replacer ad libitum $(\mathrm{n}=9)$, and milk replacer in restricted amounts $(\mathrm{n}=$ 9) were compared. Furthermore, this profiling approach was extended to the first lactation in the same animals for characterizing the long-term effect of quantitative and qualitative dietary manipulations affecting calves during development in a period that is sensitive to metabolic imprinting. Blood plasma samples were collected on d 3,22, and 52 of life as well as during wk 4 before and wk 3 and 8 after the first calving. Samples were subjected to a targeted metabolomics analysis using the AbsoluteIDQ p180 kit of Biocrates Life Science AG (Innsbruck, Austria). Profiling of metabolomics data was performed by principal component analysis and heatmap visualization of the metabolome, as well as by comparing fold changes and $t$-test statistics of metabolites. A quantitative identification of 180 plasma metabolites was possible, belonging to the metabolite classes of acyl-carnitines, AA, biogenic amines, phosphatidylcholines, lysophosphatidylcholines, sphingomyelins, and hexoses. Comparing metabolite concentrations between ad libitum-reared and restrictively reared animals revealed significant differences both during calfhood as well as during first lactation. Most dominantly, acylcarnitines of both short- and longchain length were more abundant in ad libitum reared
\end{abstract}

\footnotetext{
Received February 8, 2018.

Accepted May 7, 2018.

${ }^{1}$ Current address: College of Veterinary Medicine and Life Sciences, City University of Hong Kong, Kowloon Tong, Hong Kong SAR.

${ }^{2}$ Corresponding author: korinna.huber@uni-hohenheim.de
}

animals in the long-term, suggesting alterations in mitochondrial function, most likely indicating adaptive mechanisms of energy expenditure. Furthermore, plasma sphingomyelin concentrations were affected by ad libitum versus restricted milk replacer feeding, which can imply long-term modulatory mechanisms affecting insulin sensitivity. The functional characterization of the identified metabolic patterns, particularly the alterations of single lipid species, is required for further improving our understanding of the links between early nutrition shaping metabolic development and a healthy productive life of Holstein dairy cows.

Key words: calf nutrition, metabolic imprinting, metabolomics

\section{INTRODUCTION}

Adequate nutrition of dairy calves is considered a fundamental factor for an optimal lifetime productivity and for profitability in dairy systems (Heinrichs, 1993). Average daily gain during early age was shown to influence milk production in the first lactation (Zanton and Heinrichs, 2005). In support of this concept, several studies documented that a higher plane of nutrition during the preweaning period (i.e., ad libitum vs. restricted liquid feeding) was associated with enhanced productive performance in adult life (Shamay et al., 2005; Khan et al., 2011; Soberon et al., 2012). However, in addition to quantitative adequacy of macronutrients in liquid feed (milk or milk replacer) used for calf rearing, liquid feed quality and, particularly, presence of non-nutritive feed components such as bioactive molecules supporting neonatal development (i.e., growth factors, peptides) should be considered (Bartol et al., 2013). These molecules are present in whole milk, and calves fed whole milk ad libitum were found to produce more milk during their first lactation than calves fed milk replacer ad libitum (Moallem et al., 2010). In addition to increased milk yield, enhanced organ growth, mammary gland development, 
and gut-associated immune system development were associated with a higher plane of preweaning nutrition (Geiger et al., 2016; Wilson et al., 2017; Hammon et al., 2018). Presumably, epigenetic mechanisms play a role in maintaining long-term effects of preweaning nutrition, accounting for stable alterations in the phenotype as a form of metabolic imprinting (Bartol et al., 2013). Furthermore, early life is a critical period for shaping allometric growth and development of the mammary gland (Bach, 2012; Esselburn et al., 2015).

Previous studies reported a substantial amount of data on the links between nutrition, growth rate, and later productive performance, but less is known about the underlying metabolic pathways. In particular, we have a knowledge gap on the longitudinal changes of metabolic phenotypes at a molecular level that would extend our understanding on the differential long-term effects of preweaning nutrition. In the current study, we investigated this issue at different levels, and the present paper is the third part focusing on changes of the plasma metabolome of calves and later of the same animals as heifers. Calves were differentially reared on either whole milk ad libitum or milk replacer ad libitum or milk replacer at restricted amounts, and were followed up as heifers until their first lactation. Growth performance, production performance, and economic aspects of a higher plane of nutrition in relation to an enhanced production performance were already published as part one (Korst et al., 2017). In the second part of the study, conventionally used markers of metabolism and endocrine system in calves and heifers were analyzed (Kesser et al., 2017). However, these markers, including serum free fatty acids, BHB, glucose, insulin, adiponectin, leptin, and a surrogate index for estimating insulin sensitivity (revised quantitative insulin sensitivity check index; RQUICKI), failed to differentiate between heifers that experienced different preweaning nutrition as calves. To extend this investigation, the objective of the present paper was to perform a systemic screening of the plasma metabolome to search for acute as well as for long-lasting metabolic changes linked to the described nutrition before weaning. To do so, a targeted metabolomics approach assessing markers of basic cellular physiological and pathophysiological pathways was used to assess metabolic profiles of the animals across nutritional treatments and along the time axis. The purpose of characterizing such metabolic profiles was to visualize the dynamic development of the metabolic network influenced by nutrition-derived metabolic imprinting effects. The fundamental hypothesis of this approach was that nutrient availability and the source of nutrients (whole milk vs. milk replacer) during early life can modulate cellular physiological pathways of growth, development, maturation of im- mune and endocrine systems, as well as energy sensing and expenditure. These could collectively be defined as metabolic imprinting, as a sum of processes modulating efficiency (i.e., amount of output by utilizing the available resources) of growth, maintenance, and production. The desired optimum efficiency can be described as the maintenance of cellular processes in a healthy status and assuring an equilibrium between maintenance of body resources and production output at the same time. It might be technically challenging to identify a causative relationship between metabolic imprinting and later production outcome, but analyzing the metabolic phenotype can improve our understanding of this relationship because distinct metabolic profiles can reflect associations between nutritional treatments and production profiles.

The overall aim of the study presented in this manuscript series was to establish the potential link of metabolic imprinting deriving from rearing calves on whole milk versus milk replacer and rearing calves ad libitum versus restrictively to an altered production efficiency profile at the time when these calves develop into heifers and enter production. We evaluated the effect of liquid feed source and availability on growth performance and later production performance as well as the associated economic investment-return aspects in the first part of this series (Korst et al., 2017). In the second part, we investigated how the endocrine system was affected in these calves and heifers (Kesser et al., 2017). Finally, the aim of the currently presented third part was to characterize phenotypic patterns linked to the previously reported production profiles as a metabolic profiling approach and to generate novel hypotheses regarding molecular mechanisms of early nutrition-derived metabolic imprinting in Holstein dairy heifers.

\section{MATERIALS AND METHODS}

\section{Animals, Nutritional Treatments, and Sampling}

All animal experiments were performed in strict accordance with the German Law for the Protection of Animals and were approved by the relevant authority (Landesuntersuchungsamt Rheinland-Pfalz, Koblenz, Germany; G 11-20-026). The trials were conducted on calves and later during the first lactation of the same animals as heifers at the Educational and Research Centre for Animal Husbandry, Hofgut Neumühle, Münchweiler an der Alsenz, Germany. The effects of different feeding intensities on performance and production, as well as on metabolic and endocrine status from birth over the first lactation, were previously published (Kesser et al., 2017; Korst et al., 2017). The current study was conducted by using all female calves used in the 
aforementioned trials and using the same animals later as heifers. A detailed description of feed ingredients and nutrient composition and the gross outcome regarding performance was published by Korst et al. (2017) and is summarized below. The experimental design is graphically displayed in Supplemental Figure S1 (https://doi .org/10.3168/2018-14559).

Nutritional Interventions During Calfhood. Twenty-eight female German Holstein calves were enrolled in the present study. All calves received at least $2 \mathrm{~L}$ of colostrum milked from their dam within $2 \mathrm{~h}$ after birth. From the second feeding until d 3 of age, all calves received colostrum and transition milk ad libitum from their dam. On d 4, calves were randomly assigned to 1 of the 3 nutritional treatment groups (balanced for birth weight and parity of the dam) and from $\mathrm{d} 4$ to 27 of age they were fed accordingly. Calves of the milk replacer-restricted group (MR-res, $\mathrm{n}=$ 9) received milk replacer $\left(11.5 \%\right.$ solids; $39^{\circ} \mathrm{C}$ drinking temperature) limited to $6.78 \mathrm{~kg}$ of liquid fed/d (equals $0.78 \mathrm{~kg}$ of milk replacer powder/d); calves of the milk replacer ad libitum group (MR-adlib, $\mathrm{n}=9$ ) and the whole milk ad libitum group (WM-adlib, $\mathrm{n}=10$ ) had free access $24 \mathrm{~h} / \mathrm{d}$ to milk replacer ( $13.8 \%$ solids) or whole milk, respectively. Measured liquid feed intake between $\mathrm{d} 2$ and 27 was $6,385 \pm 39,9,249 \pm 150$, and $9,470 \pm 137 \mathrm{~g} / \mathrm{d}$ (means $\pm \mathrm{SEM}$ ) in the MR-res, MRadlib, and WM-adlib groups, respectively. The acidified milk replacer $(\mathrm{pH} 4.7)$ was provided by Trouw Nutrition Deutschland GmbH (Burgheim, Germany). The milk replacer consisted of $50 \%$ skim milk, $25 \%$ dried whey, $16.5 \%$ blend of plant oils (palm oil, coconut oil, sunflower oil), $3 \%$ glucose, $3 \%$ pregelatinized wheat flour, and $2.5 \%$ mix of trace elements and vitamins (accounting for 3,250 IU of vitamin A, $650 \mathrm{IU}$ of vitamin $\mathrm{D}_{3}, 32.5 \mathrm{mg}$ of vitamin $\mathrm{E}, 26 \mathrm{mg}$ of vitamin $\mathrm{C}, 13 \mathrm{mg}$ of iron, $1.04 \mathrm{mg}$ of copper, $15.6 \mathrm{mg}$ of zinc, $5.2 \mathrm{mg}$ of manganese, $390 \mu \mathrm{g}$ of iodine, and $45.5 \mu \mathrm{g}$ of selenium per liter of milk replacer solution). Nutrient composition of the milk replacer powder was $23.0 \% \mathrm{CP}, 17.0 \%$ crude fat, and $7.4 \%$ crude ash on a DM basis, $96.6 \%$ DM. The whole milk was saleable bulk tank milk (average $3.9 \%$ fat and $3.3 \%$ protein) from Hofgut Neumühle (Münchweiler a.d. Alsenz, Germany) and was acidified with $2 \mathrm{~mL}$ of acidifier/L of milk (Schaumacid; H. W. Schaumann GmbH, Pinneberg, Germany) to attain a $\mathrm{pH}$ of 4.6. The whole milk was supplemented with a mix of trace elements and vitamins $(1 \mathrm{~mL} / \mathrm{L}$ of Milkivit Quick-Mix, Trouw Nutrition Deutschland GmbH), which resulted in an added amount of $12 \mathrm{mg}$ of iron, $1 \mathrm{mg}$ of copper, $2.5 \mathrm{mg}$ of zinc, $2.5 \mathrm{mg}$ of manganese, $20 \mu \mathrm{g}$ of cobalt, $40 \mu \mathrm{g}$ of selenium, 4,000 IU of vitamin A, $400 \mathrm{IU}$ of vitamin $\mathrm{D}_{3}, 30 \mathrm{mg}$ of vitamin $\mathrm{E}, 7.5 \mathrm{mg}$ of vitamin $\mathrm{C}, 0.5 \mathrm{mg}$ of vitamin $\mathrm{K}_{3}, 3.0 \mathrm{mg}$ of vitamin
$\mathrm{B}_{1}, 2.0 \mathrm{mg}$ of vitamin $\mathrm{B}_{2}, 1.0 \mathrm{mg}$ of vitamin $\mathrm{B}_{6}, 8.0 \mu \mathrm{g}$ of vitamin $\mathrm{B}_{12}, 20 \mu \mathrm{g}$ of biotin, $10 \mathrm{mg}$ of niacin, $6.0 \mathrm{mg}$ of calcium-D-pantothenate, and $160 \mu \mathrm{g}$ of folic acid per liter of milk.

Birth weight was not different between the groups. For the first $7 \mathrm{~d}$ of life, all calves were kept in individual straw-bedded hutches (Flixbox, Mayer Maschinenbaugesellschaft mbH, Tittmoning, Germany) and fed twice daily by a teat bucket. From d 8 onwards, calves were housed in straw-bedded group pens with an automatic feeding system (Vario Kombi, Förster-Technik GmbH, Engen, Germany). Calves of the different groups were mixed in the pens, and differential feeding was achieved with transponder collars, through which the calves had access to their group-specific diet. All calves had free access to water, grass hay (first cut, not chopped), and concentrate.

The period of differential nutrition in the present study lasted solely from d 4 to 27 of age. From d 25 to 27, calves of the MR-adlib and the WM-adlib groups were gradually adapted (reducing the daily feed intake by $0.86 \mathrm{~kg}$ on average) to the MR-res feeding regimen (11.5\% solids, maximal daily allowance of $6.78 \mathrm{~kg}$ liquid feed), on which they continued until d 55 . Thereafter, all calves were gradually stepped down to $2 \mathrm{~kg}$ of liquid feed by d 69 (i.e., reducing daily offers by $0.34 \mathrm{~kg}$ of liquid feed). From d 70 onwards, liquid feed supply was entirely stopped and all calves had free access to a TMR (on a grass silage/corn silage/pressed beet pulp silage basis, DM $48.2 \%, 6.8 \mathrm{MJ} / \mathrm{kg}$ of DM) for lactating dairy cows. From birth until d 70, daily feed intake and energy intake values were recorded and calculated and BW was measured once weekly, as described in Korst et al. (2017).

Follow-Up as Heifers During First Lactation. The same 28 animals were used to study long-term effects of preweaning nutrition on metabolism. They were kept in a loose-housing system with high boxes and rubber mattresses, were fed a TMR for heifers (on a grass silage basis, DM $38.4 \%, 5.8 \mathrm{MJ} / \mathrm{kg}$ of $\mathrm{DM}$ ), and were AI after reaching an age of at least 15 mo. From 3 wk before expected calving date onwards they were given free access to a TMR for lactating dairy cows (on a grass silage/corn silage/pressed beet pulp silage basis, DM 44.8\%, 7.0 MJ/kg of DM).

Collection of Blood Plasma Samples. Blood samples were taken on d 3 (before start of the experimental feeding), 22 (with all 3 groups being on differential experimental feeding), and 52 (after readjustment of equal feeding conditions for all groups) from the calves by jugular venipuncture, as well as on wk 4 prepartum, wk 3 postpartum, and wk 8 postpartum at heifer age by coccygeal venipuncture. Access to feed was withdrawn $2 \mathrm{~h}$ before blood sampling. Blood samples were col- 
lected in commercially available fluoride-heparin tubes (Sarstedt AG, Nümbrecht, Germany) and were centrifuged within $30 \mathrm{~min}$ at $2,000 \times g$ for $15 \mathrm{~min}$ at $20^{\circ} \mathrm{C}$. Heparin-prepared plasma is one of the recommended specimen for the Absolute IDQ p180 metabolomics kit (Biocrates Life Science AG, Innsbruck, Austria); however, it should be noted here that systematic differences are known for a few metabolites of this kit, depending on the used blood preparation method (serum vs. EDTA plasma vs. heparin plasma; Siskos et al., 2017). Plasma was immediately frozen and stored at $-20^{\circ} \mathrm{C}$ for 1 yr until analysis. To rule out metabolite loss during storage, metabolite availability was validated by comparing metabolite concentrations detected in our study (in 3-d-old calves), with concentrations measured in plasma samples that were stored at $-80^{\circ} \mathrm{C}$ for $1 \mathrm{mo}$ after collection $(\mathrm{n}=6,3$-d-old calves from the same farm with the same nutritional and housing conditions). Storage conditions $\left(1 \mathrm{yr}\right.$ at $-20^{\circ} \mathrm{C}$ vs. $1 \mathrm{mo}$ at $-80^{\circ} \mathrm{C}$ ) were found to have no significant effect on metabolite concentrations, analyzed by principal component analysis and $t$-test.

\section{Metabolomics Analysis}

Plasma metabolome analysis was performed in all samples by the AbsoluteIDQ p180 kit (Biocrates Life Science AG). The analytical procedure was performed in the laboratory of Biocrates Life Science AG according to the manufacturer's standard protocol, as briefly presented below. This kit-format targeted metabolomics measurement was used to identify and quantify up to 188 metabolites belonging to 5 compound classes: acylcarnitines (40), proteinogenic and modified AA (19), glycerophospho- and sphingolipids (76 phosphatidylcholines, 14 lysophosphatidylcholines, 15 sphingomyelins), biogenic amines (19), and hexoses (1). A detailed list of the compounds is shown in Supplemental Table S1 (https://doi.org/10.3168/jds.2018-14559). The fully automated assay was based on phenylisothiocyanate derivatization in the presence of internal standards followed by flow injection analysis-MS/MS [acylcarnitines, (lyso-) phosphatidylcholines, sphingomyelins, hexoses] and LC-MS/MS (AA, biogenic amines) using a Sciex 4000 QTRAP (Sciex, Darmstadt, Germany) or a Xevo TQ-S Micro (Waters, Vienna, Austria) instrument with electrospray ionization. The experimental metabolomics measurement technique is described in detail by patent US 2007/0004044 (Ramsay et al., 2007). All preanalytical and analytical procedures were performed, documented, and reviewed according to the ISO (2008) certified in-house quality management rules and guidelines of Biocrates Life Sciences AG.

\section{Data Analysis and Visualization}

Plasma metabolome data (absolute concentrations of compounds) were analyzed following the same analysis pipeline as described by Kenéz et al. (2016). We analyzed the data in MetaboAnalyst 3.0 (Xia et al., 2015) after normalization by log-transformation and Pareto scaling. Principal component analysis (PCA) was used as an unsupervised method to display differences in metabolic profiles between the sampling time points and across treatments. For the following analyses, metabolome data of d 22 and 52 as well as wk $-4,3$, and 8 were all normalized to the first sampling point (d 3) to reduce noise in the data set and compare all metabolites to a uniform baseline (until d 3 no differential treatment was applied to the animals). The data set consisting of all metabolite concentrations was analyzed by repeated measures ANOVA for time effect and treatment effect, also considering interactions between the 2 and applying false discovery rate correction in MetaboAnalyst (time series +1 experimental factor design). To demonstrate which metabolites were most affected by time in calves and heifers, a heatmap of the 30 most significant metabolites (based on ANOVA time effect $P$-value) was plotted using the average values of the sampling time points separately for the 3 treatment groups. Metabolite concentrations were compared between MR-adlib versus MR-res animals separately on d 22 and 52 as well as wk $-4,3$, and 8 by $t$-test with false discovery rate (FDR) adjustment for multiple comparisons in $\mathrm{R}$ (version 3.4.0; $\mathrm{R}$ Foundation for Statistical Computing, Vienna, Austria) to identify the metabolites that were significantly affected by preweaning nutritional treatments. Volcano plots were created in $\mathrm{R}$ to show the number and distribution of significantly affected metabolites (FDR-adjusted $P$ $<0.05)$. The same way, $t$-tests were performed and volcano plots were created for comparing MR-adlib versus WM-adlib animals. For identifying the most significant metabolites shown in the volcano plots, a table of the corresponding metabolites with their fold change (FC) and FDR-corrected $P$-value ( $t$-test) was created.

Body weight in calves and heifers and milk yield in heifers were plotted in GraphPad Prism version 6.07 for Windows (GraphPad Software, La Jolla, CA). These data were previously published and discussed in part 1 and part 2 of this manuscript series (Kesser et al., 2017; Korst et al., 2017) for a larger number of calves (both female and male) and the same cohort of heifers. To remain consistent with the currently presented metabolomics data set, BW data of the currently used female calves were reanalyzed for time effect, treatment effect, and respective interactions by repeated-measures 2 -way 
ANOVA and Tukey's post-test in GraphPad Prism. This ANOVA test was also used to reanalyze BW and milk yield data of the heifers.

\section{RESULTS AND DISCUSSION}

\section{Growth and Production Performance}

The growth and production data shown here were first published in the first and second part of this series (Kesser et al., 2017; Korst et al., 2017); however, to establish adequate context they are briefly indicated and rediscussed here with a selective focus on metabolic aspects. The ad libitum fed calves (MR-adlib and WM-adlib) had greater BW during the period from d 21 to 56 of life $(P<0.05)$, as shown in Figure 1. Furthermore, the slopes of the weight development show that the ad libitum-fed calves gained increasingly more weight during the period of differential nutrition (until d 27) compared with the MR-res calves. After switching to the restricted feeding regimen for all calves (after d 27), the MR-adlib and WM-adlib calves grew slower and reached similar final weights around d 63 of life as the MR-res calves. Total ME intake (liquid feed and concentrate) during d 2 to 27 was $13.0 \pm$ $0.14,21.5 \pm 0.39$, and $26.2 \pm 0.43(P<0.01)$ and during d 28 to 69 was $23.3 \pm 0.21,21.7 \pm 0.20$, and $23.5 \pm 0.21(P=0.89)$ in the MR-res, MR-adlib, and WM-adlib calves, respectively $(\mathrm{MJ} / \mathrm{d}$, means \pm SEM; as published in Korst et al., 2017). To summarize, a higher plane of nutrition led to enhanced growth rates, but this enhancing effect was rapidly diminished once the previously provided energy surplus was no longer available. Even though calves did not differ in their BW from d 63 onward, they experienced different growth dynamics beforehand. This can be linked to differential macro- and micronutrient availability deriving from liquid feed and to compensating for a lower liquid feed availability with a higher voluntary solid feed intake (Korst et al., 2017). Thus, MR-res calves most likely experienced different dynamics of organ growth linked to altered fat, protein, carbohydrate, or mineral metabolism compared with the ad libitum-fed calves during the early postnatal period. This could trigger long-term effects due to metabolic imprinting by affecting maturation of metabolic processes (i.e., postnatal advancement of metabolism due to organ growth and related cellular hypertrophy and hyperplasia, development of endocrine regulation and immune competence, possible imprinting of energy-sensing signals). The mechanisms involved might be imprinting of appetite during the preweaning period and affected pancreatic $\beta$-cell development during young age, being a hallmark for reduced insulin sensitivity and metabolic syndrome and even influencing longevity, as described by the thrifty phenotype hypothesis in rat models and humans by Hales and Barker (2001) and Hales and Ozanne (2003).

Analyzing production performance data during first lactation revealed lower BW in heifers receiving MRadlib preweaning nutrition as calves, as shown in Figure 2A (Korst et al., 2017). Heifers from either the MR-res or the WM-adlib group maintained higher BW during the first 10 wk of lactation $(P<0.001)$. During the same period, MR-res heifers had lower milk yield than heifers receiving ad libitum nutrition in early life (Figure $2 \mathrm{~B}, P<0.02$ ). Evaluating production performance solely based on these 2 variables, WM-adlib heifers can be associated with the most desired pattern, having high milk yield and maintaining BW at the same time. Although MR-adlib heifers also had high milk yield, they experienced serious BW loss (Figure 2A), most likely due to intensive adipose mobilization. Finally, MR-res heifers did maintain high BW, but at the cost of producing less milk, demonstrating lower production efficiency. Transition dairy management aims for an optimal homeorhetic adaptation marked by a balance of well-maintained resources and an enhanced output, as stated by Bauman et al. (1985), which could be best achieved in the present study by the WM-adlib preweaning nutritional strategy.

Thus, quality and quantity of early postnatal nutrition determined later metabolic performance in Holstein dairy cows. In particular, the shift of resources into body mass maintenance or into milk production was influenced by the early diet. The MR-res heifers were programmed to maintain body mass before milk production, whereas MR-adlib heifers were programmed to produce milk irrespectively of body mass loss. Body mass loss of lactating cows due to excessive adipose mobilization is often related to production diseases (Drackley, 1999; Ingvartsen, 2006). Only WM-adlib heifers were able to maintain body mass and produce milk on a high level, indicating that a sufficient amount of whole milk during the first weeks of life is important for later health and performance in Holstein dairy cows. Not only is energy and macronutrient content of feed important for healthy heifer development, but also micronutrients, particularly biologically active molecules, which are rich in whole milk (Bartol et al., 2013). The observed differences in production profiles can be linked to fine-tuning mechanisms of specific metabolic pathways that might not be reflected by the conventionally analyzed plasma biochemical compounds in part 2 of this manuscript series (Kesser et al., 2017), but could be characterized by a comprehensive exploration of plasma metabolic profiles by metabolomics. 


\section{Metabolic Phenotypes During Early Life and First Lactation}

The metabolomics kit used in the present study was designed to quantitatively assess a defined set of metabolites reflecting a wide variety of physiological and pathophysiological cellular metabolic pathways and related metabolic disorders, such as proinflammatory status, dysregulation of glucose and lipid metabolism, obesity-related disorders, insulin resistance, and mitochondrial dysfunction. In the bovine species, this panel has been used to characterize cellular function and dysfunction, immune system and defense mechanisms, pre- and proinflammatory processes eventually coupled with tissue damage, genetic mismatch with management and production overload, as well as lack of optimum efficiency (Hailemariam et al., 2014a,b; Imhasly et al., 2014; Huber et al., 2016; Kenéz et al., 2016; Dervishi et al., 2017). The purpose of those recently published studies was to obtain a better understanding of disease development and pathobiology as well as to identify biomarkers by studying healthy and diseased conditions (such as ketosis, fatty liver disease, and retained placenta).

The results of the present metabolomics study provide insight into the gradual development of me- tabolism during early life and during first lactation using plasma samples of the same animals at multiple stages of growth and production. As shown in Figure 3, metabolic profiles, represented by the PCA scores, were completely separated between calf age and heifer age. It should be highlighted that the same animals were followed up for $2 \mathrm{yr}$ to characterize metabolic phenotypes during calfhood and during first lactation.

The metabolites that demonstrated the most significant changes depicting the massive metabolic transformation that cattle undergo between the functional monogastric state as calves and functional ruminants as heifers can be seen in Figure 4. This shows that, among the 30 most significant metabolites, several AA and the sum of hexoses (assumedly mainly glucose) were higher during calf age (especially on d 3 of life), whereas phosphatidylcholines and sphingomyelins were higher during heifer age (especially in wk 8 after calving).

The significance of this metabolomics data set lies in its long-term longitudinal and top-down systemsoriented character. The strength of metabolomics is to provide a descriptive insight into comprehensive phenotypic profiles as an integral of internal (genetic, epigenetic) and external (environment, nutrition) factors (Patti et al., 2012). It has been recognized in human metabolic research that this kind of approach,

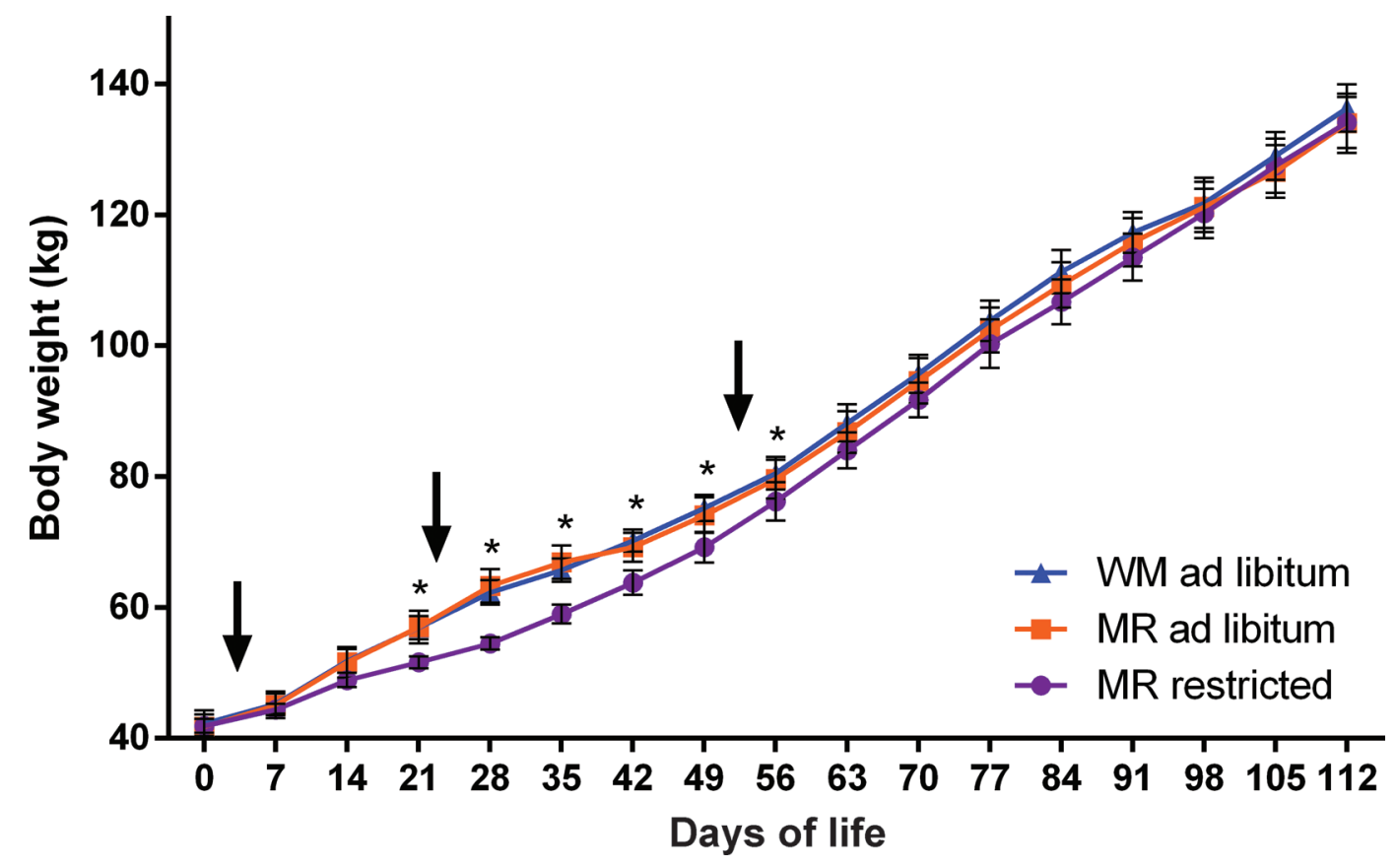

Figure 1. Body weight development of calves during the first 16 wk of age. Between d 4 and 27 of life, calves were fed whole milk (WM) ad libitum, milk replacer (MR) ad libitum, or MR restricted to $6 \mathrm{~L} / \mathrm{d}$. Calves reared on MR in restricted amounts had significantly lower live weights during d 21 to 56 than calves reared ad libitum (either on MR or WM). Repeated measures 2-way ANOVA with Tukey's post-test: time $=P<0.001$, diet $=P<0.001$, interaction $=P<0.001$; and asterisk $(*)$ indicates $P<0.05$ for MR restricted vs. MR ad libitum and MR restricted vs. WM ad libitum on marked days. Arrows indicate times of blood sampling. Shown are group means \pm SEM, $\mathrm{n}=9$ (for MR ad libitum and MR restricted) and $n=10$ (for WM ad libitum). Altered growth dynamics linked to differential nutrient availability or conversion rate might trigger long-term metabolic effects and affect metabolic health and longevity. Color version available online. 


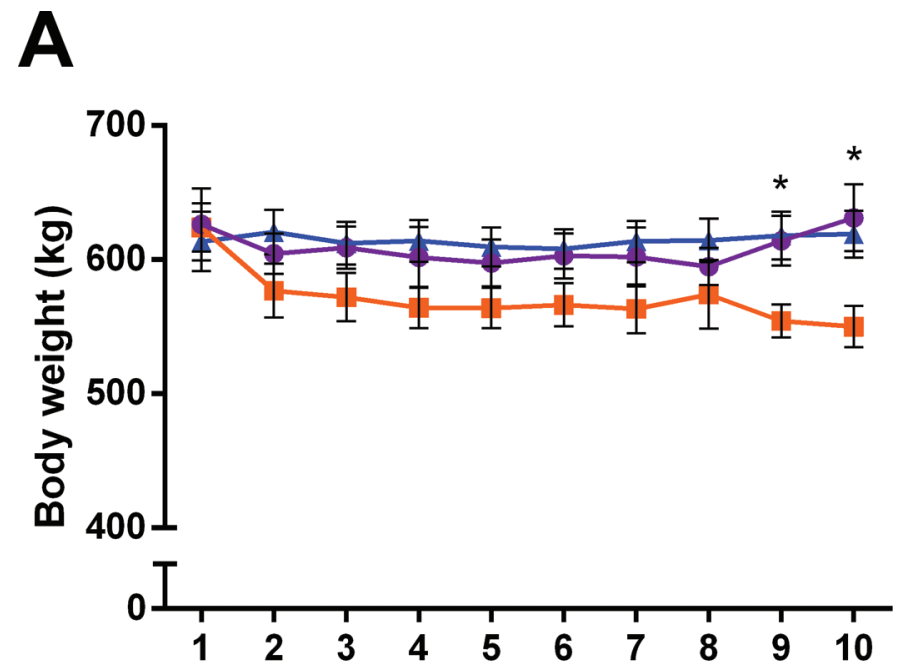

B

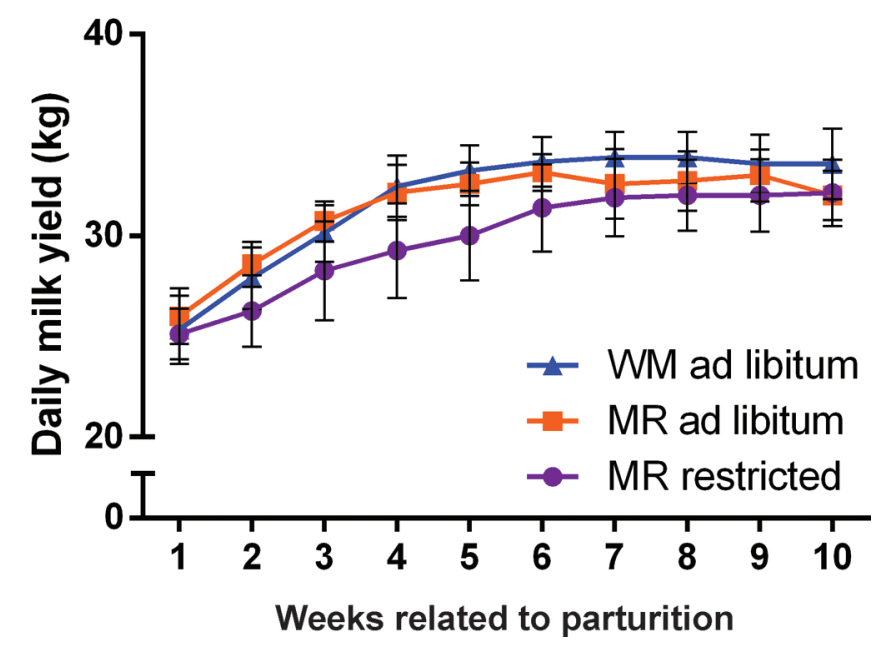

Figure 2. (A) Body weight and (B) daily milk yield in heifers during first $10 \mathrm{wk}$ of lactation. Between d 4 and 27 of life, calves were fed whole milk (WM) ad libitum, milk replacer (MR) ad libitum, or MR restricted to $6 \mathrm{~L} / \mathrm{d}$. Deriving from this longitudinal study, data are shown for heifers receiving different preweaning diet during early life as calves (WM ad libitum, MR ad libitum, or MR in restricted amounts). Repeated measures 2-way ANOVA with Tukey's post-test: (A) $\mathrm{BW}$ : time $=P=0.95$, diet $=P<0.001$, interaction $=P=0.99$; (B) milk yield: time $=P<0.001$, diet $=P=0.02$, interaction $=P$ $=0.99$. An asterisk $\left(^{*}\right)$ indicates $P<0.05$ for $\mathrm{MR}$ ad libitum vs. MR restricted and $\mathrm{MR}$ ad libitum vs. WM ad libitum on marked days. Shown are group means \pm SEM, $\mathrm{n}=9$ (for MR ad libitum and MR restricted) and $\mathrm{n}=10$ (for $\mathrm{WM}$ ad libitum). Color version available online.

exploring longitudinal influence of early life nutrition and lifestyle, opens up new research areas addressing (patho-) physiological processes leading to metabolic function and dysfunction during adult age (Moco et al., 2013). However, it has to be noted that the present data set did not intend to identify biomarkers, because this would require a greater number of biological replicates.
Instead, our data set intends and allows for creating new hypotheses about metabolic programming that can serve as a basis for further testing. In calves it was previously documented that nutritional and environmental factors are linked to later productive performance (Shamay et al., 2005; Zanton and Heinrichs, 2005; Moallem et al., 2010; Soberon et al., 2012); however, to understand the underlying physiological mechanisms in greater detail, more data on the long-term metabolic development associated with distinct growth and production performance levels are needed. Metabolomics provides a useful tool to achieve this, but only a limited amount of metabolomics data in calves are available (focusing mainly on infectious diseases; Basoglu et al., 2016). The descriptive presentation of metabolic profiles seen in Figure 3 gives a basic impression on the dominance of the time effect that shapes metabolic development in calves, with a less remarkable separation according to the differential feeding and consequent differences in growth performance. The high AA and hexose (glucose) concentration during early neonatal life was anticipated, in line with the rapidly decreasing dietary availability of these nutritive components with time. As expected, separated by a large time span, metabolic profiles of heifers did not show any overlap with the calves' profiles; however, they also exhibited a time-related shift around calving, as previously observed in periparturient multiparous cows (Kenéz et al., 2016). Compared with calves, heifers had remarkably higher concentrations of phosphatidylcholines and sphingomyelins. Currently, these groups of lipid moieties are triggering increasing research interest because they are often linked to physiological and pathophysiological regulation. Even though many details of the exact biological function associated with the single lipid molecules belonging to these compound classes are not clear yet, they were recently often associated with cellular regulation of inflammatory processes, energy homeostasis, and insulin sensitivity (Gault et al., 2010; Imhasly et al., 2015; Rico et al., 2015).

\section{Ad Libitum versus Restricted Availability of Milk Replacer}

Comparing metabolite concentrations between MRadlib and MR-res animals revealed significant differences, predominantly during heifer age. In calves, only 1 of the acylcarnitines, hexadecenoyl carnitine (C16:1), was found to be significantly different (FRD-corrected $P<0.05, t$-test) on d 22, as shown in the volcano plots combining FC and $P$-value statistics in Figure 5A. This reflects an acute effect of the feeding regimens, with $\mathrm{d}$ 22 being a time point where calves actually were receiving different quantities of milk replacer and already 


\section{PCA Scores Plot}

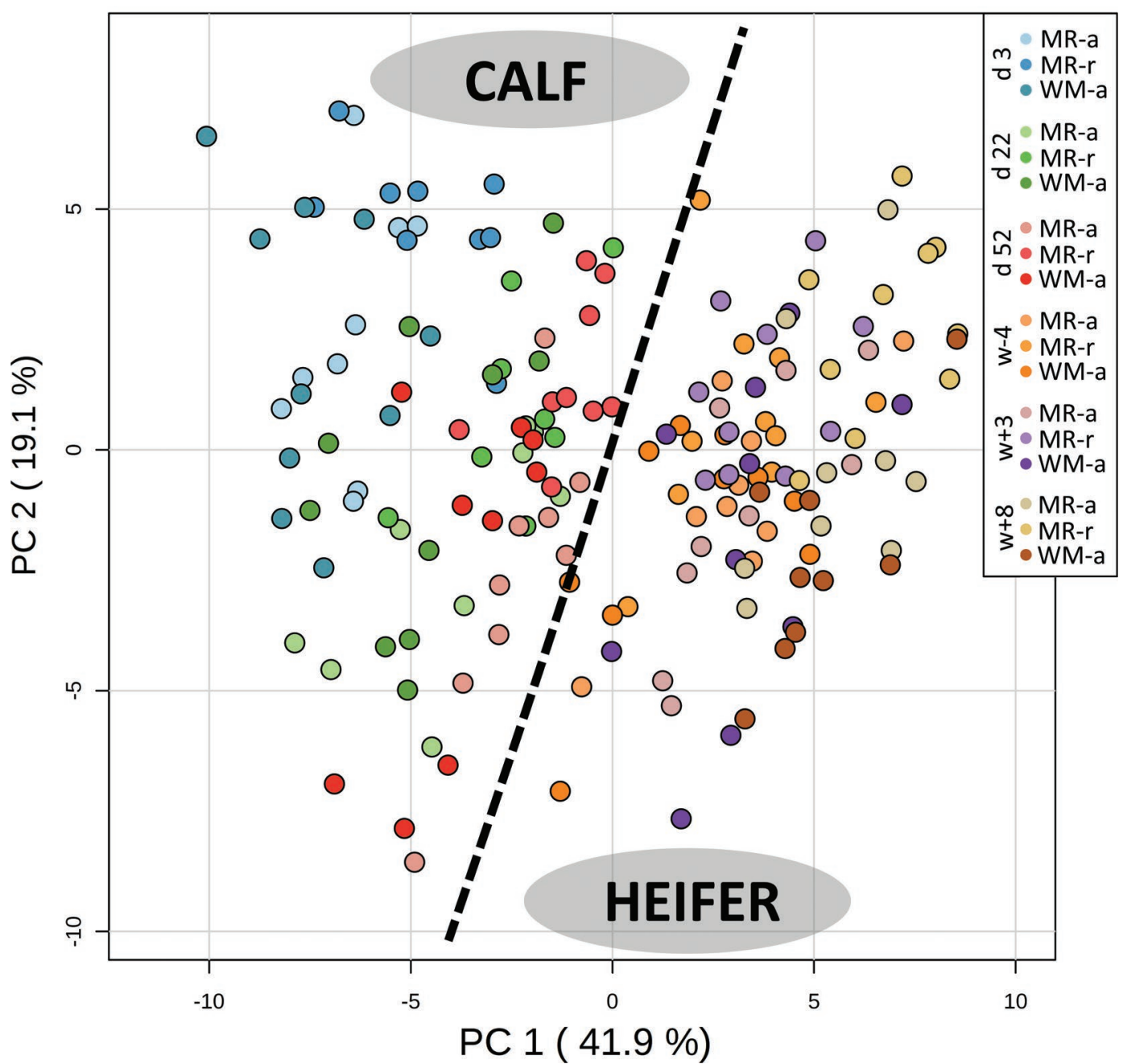

Figure 3. Principal component analysis (PCA) of plasma metabolome data during early life (d 3, 22, and 52 of life) and around first calving (wk $-4,3$, and 8 related to calving). In this longitudinal study from birth to first lactation, the plasma metabolome (180 metabolites) of calves and heifers was analyzed. Calves were fed whole milk ad libitum (WM-a; $\mathrm{n}=10$ ), milk replacer ad libitum $(\mathrm{MR}-\mathrm{a} ; \mathrm{n}=9)$, or milk replacer restricted to $6 \mathrm{~L} / \mathrm{d}(\mathrm{MR}-\mathrm{r} ; \mathrm{n}=9)$ between $\mathrm{d} 4$ and 27 of life. The PCA scores of individual animals assigned to different preweaning nutrition (rearing on MR-a, MR-r, or WM-a) are shown in different shades. Different colors indicate different sampling time (d 3, 22, and 52, wk -4, 3, and 8). The most dominant effects seen on the PCA scores plot is the complete separation of metabolic profiles between calf age and heifer age (indicated by the dashed black line). An ongoing shift of the metabolic profile with increasing age in calves and during the peripartum period in heifers can be observed along the $\mathrm{x}$-axis (blue $\rightarrow$ green $\rightarrow$ red; orange $\rightarrow$ purple $\rightarrow$ brown). Color version available online.

showing increasing differences in growth rate (Figure 1). As acylcarnitines are involved in mitochondrial transport of fatty acids, a rise in C16:1 acylcarnitine in MR-adlib calves indicated an acute link between milk replacer availability and mitochondrial function. Moreover, to follow the progressing dynamics of nutrition and growth, d 52 was a time point where both groups were fed the same restricted diet and MR-adlib calves already had a decelerated growth rate due to the reduced energy intake (Figure 1). At this phase of the study, at d 52, no significant metabolites were identified between MR-adlib and MR-res.
Confirming our hypothesis regarding long-lasting metabolic effects of preweaning nutrition, we could identify several metabolites with different abundance in heifers deriving from the MR-adlib and MR-res groups. We found 26, 24, and 17 metabolites out of 180 that were different (FDR-corrected $P<0.05, t$-test) at wk $-4,3$, and 8 , respectively (Figure 5A). Table 1 lists those affected metabolites that were significantly different at any of the studied time points. These metabolites were consistent with the higher C16:1 acylcarnitine concentrations found in calves of the MR-adlib group, as several acylcarnitines were also found to be higher in 


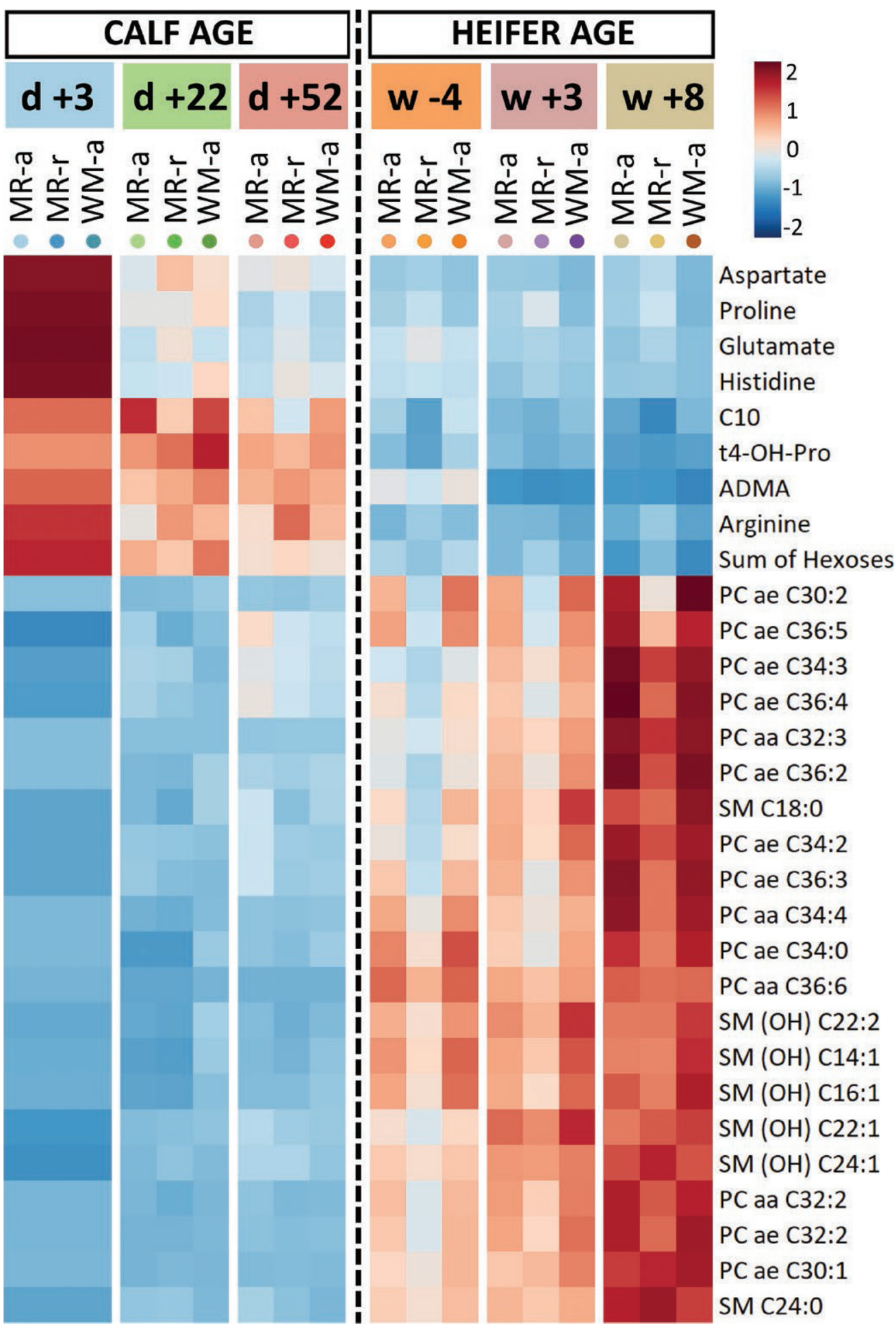

Figure 4. Heatmap visualizing the most significant metabolic changes during early life (d 3, 22, and 52 of life) and around first calving (wk $-4,3$, and 8 related to calving). In this longitudinal study from birth to first lactation, the plasma metabolome (180 metabolites) of calves and heifers was analyzed. Calves were fed whole milk ad libitum (WM-a; $\mathrm{n}=10)$, milk replacer ad libitum $(\mathrm{MR}$-a; $\mathrm{n}=9)$ or milk replacer restricted to $6 \mathrm{~L} / \mathrm{d}(\mathrm{MR}-\mathrm{r} ; \mathrm{n}=9)$ between $\mathrm{d} 4$ and 27 of life. Normalized metabolite concentrations were averaged within sampling time points for the 3 treatment groups. The 30 most significant metabolites (based on repeated measures 2 -way ANOVA, $P$-value for factor time) demonstrating increase (in red) or decrease (in blue) during time [d 3, d 22, d 52 of life, week (w) $-4, \mathrm{w}+3, \mathrm{w}+8$ relative to first calving] are shown. Several $\mathrm{AA}$ and the sum of hexoses were higher during calf age, whereas many lipid moieties were higher during heifer age. C10 = decanoylcarnitine; t4-OH-Pro = trans-4-hydroxyproline; ADMA = asymmetric dimethylarginine; $\mathrm{PC}=$ phosphatidylcholine; $\mathrm{SM}=\mathrm{sphingomyelin}$ (for a detailed list of specific PC and SM moieties, see Supplemental Table S1; https://doi.org/10.3168/jds.2018-14559). Color version available online. 
A

d 22

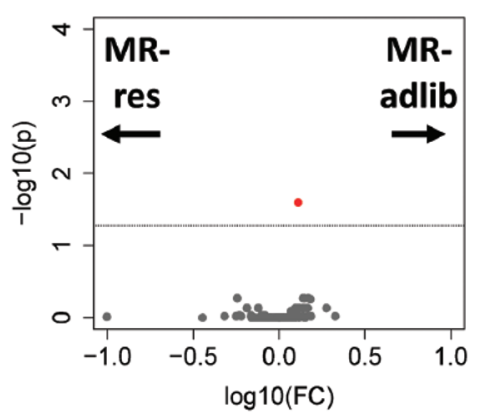

$w-4$

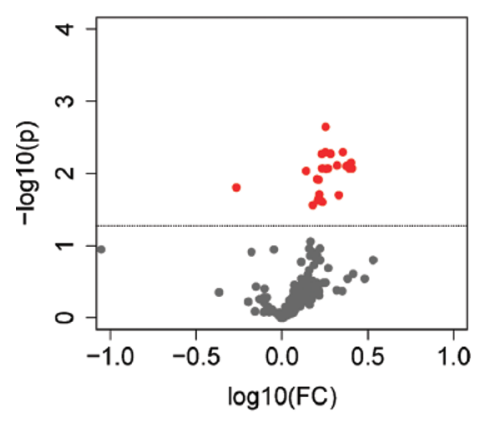

B

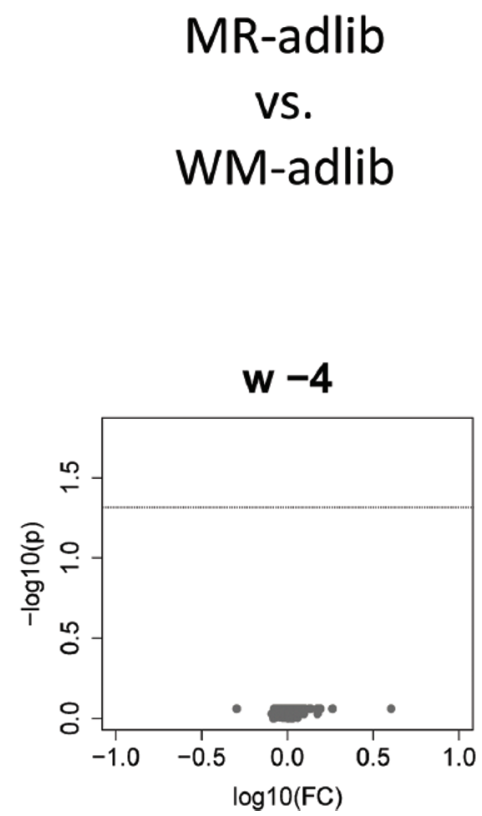

$w+3$

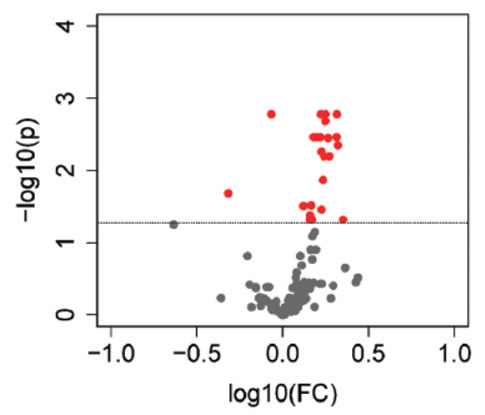

d 22
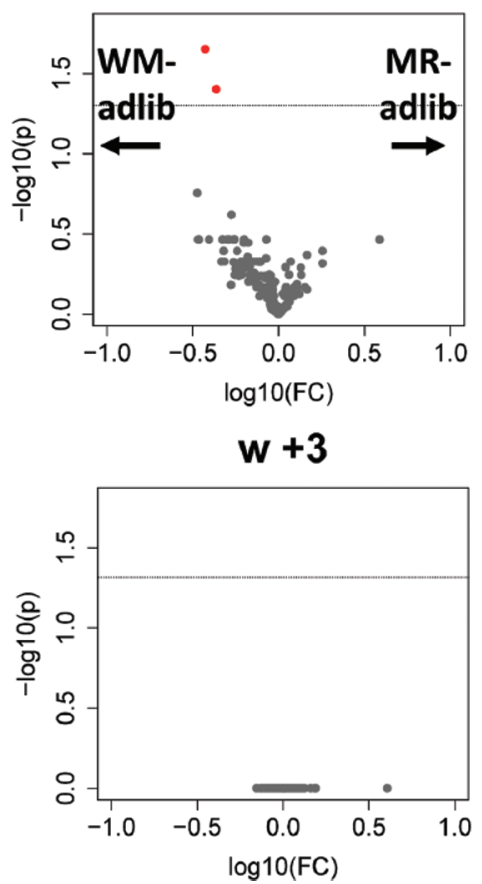

d 52

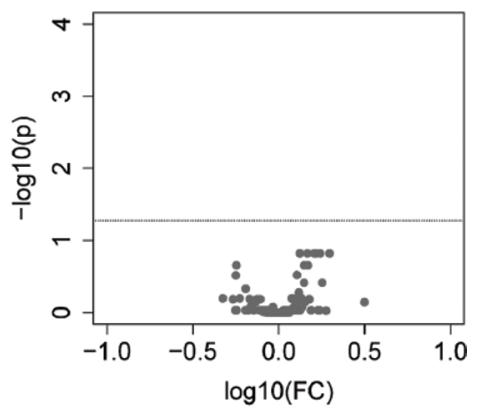

w +8

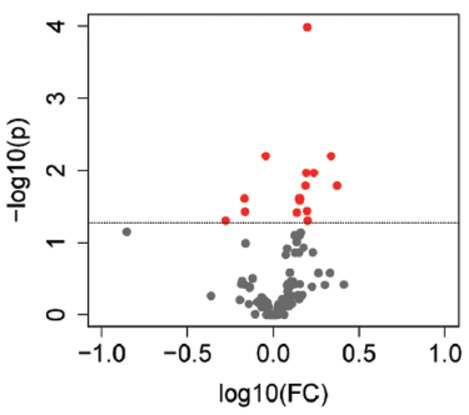

d 52
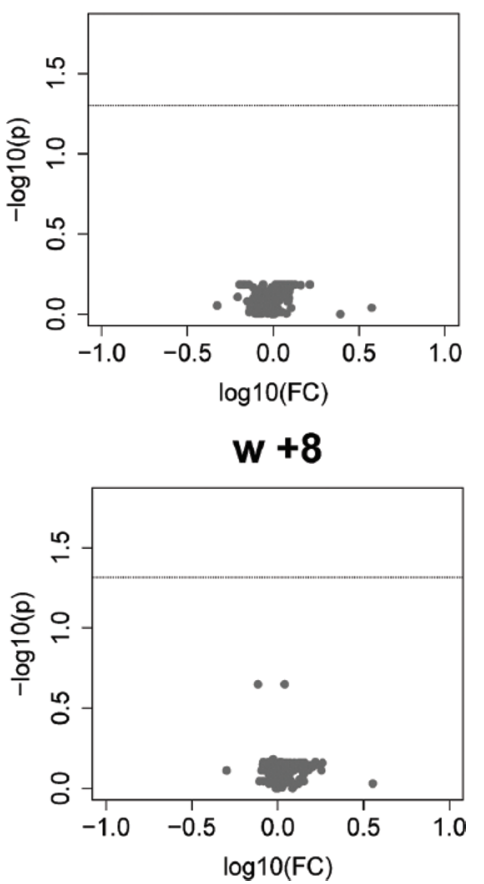

Figure 5. Volcano plots presenting the fold change (FC) and $t$-test $P$-value of metabolites and indicating significant differences (false discovery rate-adjusted $P<0.05, t$-test, red symbols above the line) between the compared nutritional treatments during early life (d 3,22 , and 52 of life) and around first calving (wk $-4,3$, and 8 related to calving). In this longitudinal study from birth to first lactation, the plasma metabolome (180 metabolites) of calves and heifers was analyzed. Calves were fed either whole milk ad libitum (WM-adlib; $\mathrm{n}=10$ ), milk replacer ad libitum (MR-adlib; $\mathrm{n}=9$ ), or milk replacer restricted to $6 \mathrm{~L} / \mathrm{d}$ (MR-res; $\mathrm{n}=9$ ) between $\mathrm{d} 4$ and 27 of life. (A) MR-adlib vs. MR-res: Red symbols in the upper right half of the plots are metabolites that had a greater concentration in the MR-adlib group compared with the MR-res group. Note that several significant metabolites were identified at the time around first calving (wk $-4,3$, and 8; indicated as symbols above the line). (B) MR-adlib vs. WM-adlib: Red symbols in the upper left half of the plots are metabolites that had a greater concentration in the WM-adlib group compared with the MR-adlib group. Note that only a few metabolites were found to be significant and this was restricted to the time of differential rearing period (d 22). Color version available online. 
MR-adlib heifers than in MR-res heifers. Furthermore, MR-adlib heifers had greater circulating concentrations of the phosphatidylcholines C30:2 and lysophosphatidylcholines C24:0 as well as of the sphingomyelin C26:1. Two other sphingomyelin compounds, sphingomyelin C18:1 and C24:1, as well as $\alpha$-aminoadipic acid were lower in MR-adlib heifers than in MR-res heifers. These findings underline that quantitative differences of the provided milk replacer not only acutely modulated metabolism during the rearing period but also affected development in calves leading to detectable alterations of metabolism even during the first lactation. The underlying mechanism might be linked to the altered growth dynamics, which not only affected organ growth but also development and imprinting of various metabolic and endocrine systems (Blum et al., 1985; Yambayamba et al., 1996).

The most prominent difference between MR-adlib and MR-res, both in calf and in heifer age, was the lower concentration of acylcarnitines in the MR-res group. Acylcarnitines are functionally associated with intracellular fatty acid transport from the cytoplasm into the mitochondria for oxidation to generate energy. Plasma acylcarnitines are known to reflect diet-dependent efflux rates that are determined by fatty acid oxidation pathways mainly in liver and skeletal muscle (Schooneman et al., 2013). Acylcarnitine concentrations were predominantly affected on the long-term in heifers (both pre- and postpartum), and the fact that short-, middle-, and long-chain acylcarnitines were consistently involved indicates a systematic effect on mitochondrial function. This persistent shift in plasma acylcarnitine profile is most likely related to long-term mitochondrial adaptation, due to epigenetic mechanisms in a similar way as described for human obesity and type 2 diabetes (Taylor et al., 2014), or due to endocrine factors modulating lipid metabolism such as leptin resistance described for metabolic syndrome (Jousse et al., 2011). Nevertheless, the current data only support a descriptive recognition of phenotypic patterns, providing a basis for the generation of new hypotheses. To clarify the mechanistic details of how mitochondrial transport mechanisms are differentially affected in the longterm, future studies are warranted. Furthermore, the relevance of distinct mitochondrial functional profiles for health status remains ambiguous. Having greater plasma acylcarnitine concentrations was previously associated with a healthier mitochondrial metabotype and longevity in dairy cows due to active clearance of excess lipid load (Huber et al., 2016). However, besides this detoxifying mechanism that permits mitochondrial efflux of overabundant acylcarnitines from tissues such as liver (Ramsay, 2000), high plasma acylcarnitines can also be interpreted as a sign for mitochondrial stress as- sociated with depleted oxidative capacity and resulting in a passive leakage of intermediary products into the circulation (Koves et al., 2008).

Furthermore, the affected lipid moieties of the phosphatidylcholines, lysophosphatidylcholines, and sphingomyelins compound classes were described as undergoing a strong decrease during times of metabolic stress associated with parturition and early lactation (Kenéz et al., 2016). Some of these compounds were also associated with incidence of metabolic disorders (Imhasly et al., 2014, 2015) and with insulin resistance (Rico et al., 2015) in dairy cows. Nevertheless, further research is needed for identifying the exact pathophysiological and signaling function assigned to specific lipid molecules in dairy cows.

\section{Ad Libitum Milk Replacer- versus Whole Milk-Based Rearing}

Comparing the metabolic profiles of calves either reared with milk replacer or with whole milk revealed differences regarding the period of the differential feeding. On d 22 of age, 2 of the measured 180 metabolites were found to be different (FDR-corrected $P<$ $0.05, t$-test) between MR-adlib and WM-adlib calves, whereas on d 52 no significantly different metabolites were found (Figure 5B). During the first lactation, metabolic profiles were found to be similar, with no significantly different metabolites affected in the long-term. This suggests that the differences seen in BW between these heifers were either not associated with distinct metabolic profiles or that the underlying metabolic alterations were not reflected in the applied targeted metabolic analysis. The present data suggest that a high plane of preweaning nutrition (i.e., ad libitum availability of high-quality liquid feed) can lead to similar metabolic profiles in the long-term, irrespective of the liquid feed source (whole milk vs. milk replacer). However, as MR-adlib heifers were found to lose more BW postpartum than WM-adlib heifers (Figure $2 \mathrm{~A}$ ), and previous studies demonstrated long lasting metabolic and endocrine effects of whole milk feeding (Moallem et al., 2010; Bartol et al., 2013), it seems to be unlikely that metabolic profiles remained fully unaffected by milk replacer- versus whole milk-based rearing. Further research is warranted to explore the potential involvement of metabolic pathways beyond the ones that are included in the presently used metabolomics assay, such as pathways driving fat deposition and endocrine interactions between mammary fad pad and mammary gland development (Moallem et al., 2010), as well as associations between epigenetic mechanisms and female reproductive function (Bartol et al., 2013). 


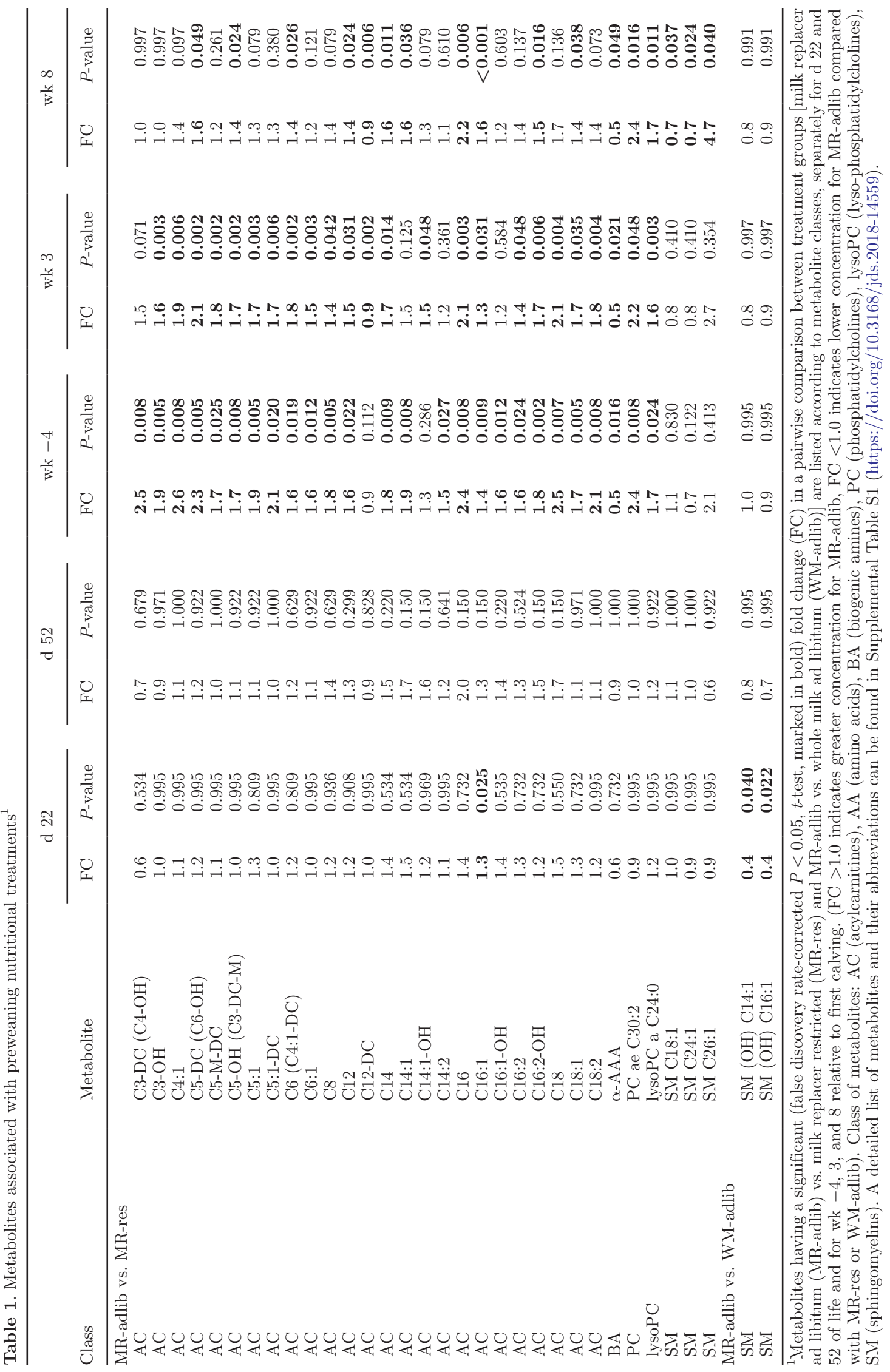


As listed in Table 1, the affected metabolites in these calves were sphingomyelins (SM OH C14:1 and SM OH C16:1), having significantly lower concentrations in the MR-adlib calves compared with WM-adlib calves on d 22. Finding significantly different metabolites only on d 22 suggests that an altered metabolic profile was induced directly or indirectly by acutely absorbed components of the liquid feed. These might include fat, protein, and carbohydrate components that were of different source when comparing milk replacer versus whole milk, as well as maternally derived bioactive molecules present in whole milk but missing in milk replacer, such as certain lipid species with a signaling function (Bartol et al., 2013). Furthermore, the difference in vitamin and trace element composition between milk replacer and whole milk has to be mentioned as a potential influencing factor too. The correct functional interpretation of the changed sphingomyelin profile requires further research, as data availability on the exact function of these compounds in preweaning calves is limited. Available data from periparturient cows show that the concentration of these sphingomyelins decreases in times of metabolic stress during early lactation (Kenéz et al., 2016), coinciding with an increase in ceramide concentrations, presumably due to a higher sphingomyelinase activity, which is associated with reduced insulin sensitivity (Rico et al., 2015). Whether the ratio between sphingomyelins and ceramides plays a role in metabolic programing in calves should be investigated in future studies.

\section{Overall Study Outcome (Summary of Part 1, Part 2, and Part 3)}

This manuscript series investigated how preweaning nutrition (i.e., source and availability of liquid feed) affects growth and metabolism of calves and how these dietary factors that affect calves in a sensitive time frame of their development affect production performance and metabolism in the long-term when these calves grow into heifers and enter their first lactation. Ad libitum feeding of milk replacer or whole milk accelerated BW gain during the actual time of ad libitum feeding (d 4-27 of life), but this benefit, compared with the restrictively fed calves, was not further maintained when the ad libitum feeding was discontinued. From an economic point of view, ad libitum feeding was associated with higher costs during the rearing period than restricted feeding, but the greater milk yield in the ad libitum-reared heifers still accounted for positive returns by the end of the first lactation (the returns over rearing feed costs in WM-adlib heifers were found to be 2.5 and $0.6 \%$ above returns in MR-res and MR-adlib heifers, respectively, as published in Korst et al., 2017).
The second and third part of the study investigated the underlying hormonal and metabolic aspects of heifer growth and development, focusing on differential longitudinal patterns as a function of preweaning nutrition. The key findings of the study across the 3 parts of this manuscript series are summarized in Figure 6. Conventional metabolites (glucose, nonesterified fatty acids, and BHB) and hormones were found to be mainly affected during the period of ongoing dietary treatments (d 4-27 of life). Among these effect, serum insulin concentration was one of the most affected, being significantly lower in restrictively fed calves than in ad libitum fed calves. However, no significant longterm effects on conventional metabolites or hormones could be confirmed in lactating heifers that could have originated from the different preweaning nutritional regimens. In contrast to the conventional metabolites and hormones, the used metabolomics approach did detect long-term differences in plasma metabolomic profiles of heifers, particularly reflecting alterations of mitochondrial function associated with ad libitum versus restricted milk replacer-based rearing. This also highlights the potential benefit of applying metabolomics as a tool to acquire a more detailed picture of the actual state of various metabolic pathways.

\section{CONCLUSIONS}

Restricted availability of milk replacer-induced alterations in the calf metabolism were persistently maintained for at least $2 \mathrm{yr}$, until the first lactation period. In particular, acylcarnitines (both short- and longchain) were found to be affected, suggesting long-term mitochondrial adaptation and, consequently, an altered metabolic profile of energy expenditure. These findings extend the previously documented relationship between quantitative nutrient availability in early age and production performance in later life. Moreover, providing greater quantities of liquid feed in form of whole milk to calves was associated with the desired production profile of greater milk yield while maintaining body resources during first lactation. The corresponding metabolic phenotype indicated involvement of mitochondrial adaptation to early life nutrient availability, reflected by alterations in plasma acylcarnitine profile. In line with recent studies focusing on metabolic flexibility and metabolic dysregulation in farm animals, the current investigation also identified phosphatidylcholines, lysophosphatidylcholines, and sphingomyelins as significant metabolite groups being altered by preweaning nutritional interventions. As these metabolite groups are structurally and functionally diverse and the (patho-) physiological function of the single lipid moieties belonging to these groups are not well understood yet, 


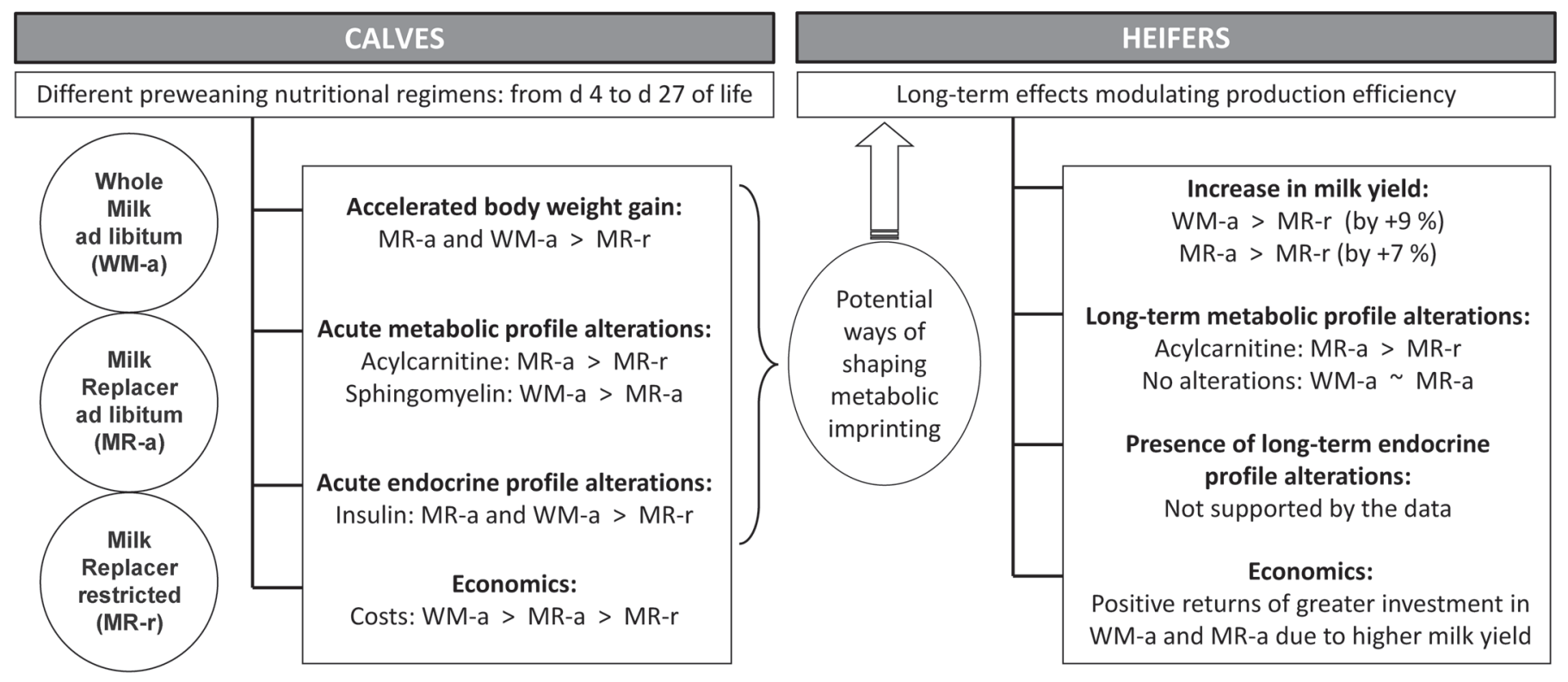

Figure 6. Graphical summary of the overall outcome of part 1, part 2, and part 3 of this manuscript series. This longitudinal study, from birth to first lactation, assessed heifer growth and development based on performance, endocrine, and metabolomics profile parameters. Calves were fed whole milk ad libitum (WM-a; $\mathrm{n}=10)$, milk replacer ad libitum (MR-a; $\mathrm{n}=9$ ), or milk replacer restricted to $6 \mathrm{~L} / \mathrm{d}(\mathrm{MR}-\mathrm{r} ; \mathrm{n}=9)$ between d 4 and 27 of life. Preweaning nutritional interventions acutely affected growth, plasma lipid profiles, and serum insulin concentrations, which represent potential ways of metabolic imprinting leading to long-term modulation of production efficiency during first lactation. This was supported by alterations in plasma acylcarnitine profiles and in milk yield, associated with differential preweaning nutrition.

future research should be carried out to clarify mechanistic relationships of these metabolites with metabolic health in dairy cows. Consistent with previous studies, our results show that investing into enhanced plane of nutrition of calves should be emphasized and further investigated, because early nutritional interventions can potentially modulate future milk production through currently poorly understood metabolic processes.

\section{ACKNOWLEDGMENTS}

The authors acknowledge the staff of the Educational and Research Centre for Animal Husbandry, Hofgut Neumühle (Münchweiler an der Alsenz, Germany).

\section{REFERENCES}

Bach, A. 2012. Ruminant nutrition symposium: Optimizing performance of the offspring: Nourishing and managing the dam and postnatal calf for optimal lactation, reproduction, and immunity. J. Anim. Sci. 90:1835-1845. https://doi.org/10.2527/jas.2011 -4516 .

Bartol, F. F., A. A. Wiley, D. J. Miller, A. J. Silva, K. E. Roberts, M. L. P. Davolt, J. C. Chen, A.-L. Frankshun, M. E. Camp, K. M. Rahman, J. L. Vallet, and C. A. Bagnell. 2013. Lactation biology symposium: Lactocrine signaling and developmental programming. J. Anim. Sci. 91:696-705. https://doi.org/10.2527/jas.2012-5764.

Basoglu, A., N. Baspinar, L. Tenori, A. Vignoli, and R. Yildiz. 2016. Plasma metabolomics in calves with acute bronchopneumonia. Metabolomics 12. https://doi.org/10.1007/s11306-016-1074-x.
Bauman, D. E., S. N. McCutcheon, W. D. Steinhour, P. J. Eppard, and S. J. Sechen. 1985. Sources of variation and prospects for improvement of productive efficiency in the dairy cow: A review. J. Anim. Sci. 60:583-592. https://doi.org/10.2527/jas1985.602583x.

Blum, J. W., W. Schnyder, P. L. Kunz, A. K. Blom, H. Bickel, and A. Schürch. 1985. Reduced and compensatory growth: endocrine and metabolic changes during food restriction and refeeding in steers. J. Nutr. 115:417-424.

Dervishi, E., G. Zhang, R. Mandal, D.S. Wishart, and B.N. Ametaj. 2017. Targeted metabolomics: New insights into pathobiology of retained placenta in dairy cows and potential risk biomarkers. Animal 12:1050-1059. https://doi.org/10.1017/S1751731117002506.

Drackley, J. K. 1999. Biology of dairy cows during the transition period: The final frontier? J. Dairy Sci. 82:2259-2273. https://doi .org/10.3168/jds.S0022-0302(99)75474-3.

Esselburn, K. M., T. M. Hill, H. G. Bateman, F. L. Fluharty, S. J. Moeller, K. M. O'Diam, and K. M. Daniels. 2015. Examination of weekly mammary parenchymal area by ultrasound, mammary mass, and composition in Holstein heifers reared on 1 of 3 diets from birth to 2 months of age. J. Dairy Sci. 98:5280-5293. https:// doi.org/10.3168/jds.2014-9061.

Gault, C. R., L. M. Obeid, and Y. A. Hannun. 2010. An Overview of Sphingolipid Metabolism: From Synthesis to Breakdown. Springer, New York, NY.

Geiger, A. J., C. L. M. Parsons, R. E. James, and R. M. Akers. 2016. Growth, intake, and health of Holstein heifer calves fed an enhanced preweaning diet with or without postweaning exogenous estrogen. J. Dairy Sci. 99:3995-4004. https://doi.org/10.3168/jds .2015-10405.

Hailemariam, D., R. Mandal, F. Saleem, S. M. Dunn, D. S. Wishart, and B. N. Ametaj. 2014a. Identification of predictive biomarkers of disease state in transition dairy cows. J. Dairy Sci. 97:2680-2693. https://doi.org/10.3168/jds.2013-6803.

Hailemariam, D., R. Mandal, F. Saleem, S. M. Dunn, D. S. Wishart, and B. N. Ametaj. 2014b. Metabolomics approach reveals altered plasma amino acid and sphingolipid profiles associated with 
pathological state in transition dairy cows. Curr. Metabolomics $2: 184-195$.

Hales, C. N., and D. J. Barker. 2001. The thrifty phenotype hypothesis. Br. Med. Bull. 60:5-20.

Hales, C. N., and S. E. Ozanne. 2003. The dangerous road of catchup growth. J. Physiol. 547:5-10. https://doi.org/10.1111/jphysiol 2002.00005.x.

Hammon, H. M., D. Frieten, C. Gerbert, C. Koch, G. Dusel, R. Weikard, and C. Kühn. 2018. Different milk diets have substantial effects on the jejunal mucosal immune system of pre-weaning calves, as demonstrated by whole transcriptome sequencing. Sci. Rep. 8 . https://doi.org/10.1038/s41598-018-19954-2.

Heinrichs, A. J. 1993. Raising dairy replacements to meet the needs of the 21st century. J. Dairy Sci. 76:3179-3187. https://doi.org/10 .3168/jds.S0022-0302(93)77656-0.

Huber, K., S. Dänicke, J. Rehage, H. Sauerwein, W. Otto, U. RolleKampczyk, and M. von Bergen. 2016. Metabotypes with properly functioning mitochondria and anti-inflammation predict extended productive life span in dairy cows. Sci. Rep. 6:24642. https://doi .org/10.1038/srep24642.

Imhasly, S., C. Bieli, H. Naegeli, L. Nyström, M. Ruetten, and C. Gerspach. 2015. Blood plasma lipidome profile of dairy cows during the transition period. BMC Vet. Res. 11. https://doi.org/10.1186/ s12917-015-0565-8.

Imhasly, S., H. Naegeli, S. Baumann, M. von Bergen, A. Luch, H. Jungnickel, S. Potratz, and C. Gerspach. 2014. Metabolomic biomarkers correlating with hepatic lipidosis in dairy cows. BMC Vet. Res. 10:122.

Ingvartsen, K. L. 2006. Feeding- and management-related diseases in the transition cow. Anim. Feed Sci. Technol. 126:175-213. https:// doi.org/10.1016/j.anifeedsci.2005.08.003.

ISO. 2008. 9001:2008 Quality management systems. Requirements International Organization for Standardization (ISO), Geneva, Switzerland.

Jousse, C., L. Parry, S. Lambert-Langlais, A.-C. Maurin, J. Averous, A. Bruhat, V. Carraro, J. Tost, P. Letteron, P. Chen, R. Jockers, J.-M. Launay, J. Mallet, and P. Fafournoux. 2011. Perinatal undernutrition affects the methylation and expression of the leptin gene in adults: Implication for the understanding of metabolic syndrome. FASEB J. 25:3271-3278. https://doi.org/10.1096/fj.11 -181792 .

Kenéz, Á., S. Dänicke, U. Rolle-Kampczyk, M. von Bergen, and K. Huber. 2016. A metabolomics approach to characterize phenotypes of metabolic transition from late pregnancy to early lactation in dairy cows. Metabolomics 12. https://doi.org/10.1007/s11306-016 $-1112-8$.

Kesser, J., M. Korst, C. Koch, F.-J. Romberg, J. Rehage, U. Müller, M. Schmicke, K. Eder, H. M. Hammon, H. Sadri, and H. Sauerwein. 2017. Different milk feeding intensities during the first 4 weeks of rearing dairy calves: Part 2: Effects on the metabolic and endocrine status during calfhood and around the first lactation. J. Dairy Sci. 100:3109-3125. https://doi.org/10.3168/jds.2016-11595.

Khan, M. A., D. M. Weary, and M. A. G. von Keyserlingk. 2011. Invited review: Effects of milk ration on solid feed intake, weaning, and performance in dairy heifers. J. Dairy Sci. 94:1071-1081. https://doi.org/10.3168/jds.2010-3733.

Korst, M., C. Koch, J. Kesser, U. Müller, F.-J. Romberg, J. Rehage, K. Eder, and H. Sauerwein. 2017. Different milk feeding intensities during the first 4 weeks of rearing in dairy calves: Part 1: Effects on performance and production from birth over the first lactation. J. Dairy Sci. 100:3096-3108. https://doi.org/10.3168/ jds.2016-11594.

Koves, T. R., J. R. Ussher, R. C. Noland, D. Slentz, M. Mosedale, O. Ilkayeva, J. Bain, R. Stevens, J. R. B. Dyck, C. B. Newgard, G. D. Lopaschuk, and D. M. Muoio. 2008. Mitochondrial overload and incomplete fatty acid oxidation contribute to skeletal muscle insulin resistance. Cell Metab. 7:45-56. https://doi.org/10.1016/j .cmet.2007.10.013.

Moallem, U., D. Werner, H. Lehrer, M. Zachut, L. Livshitz, S. Yakoby, and A. Shamay. 2010. Long-term effects of ad libitum whole milk prior to weaning and prepubertal protein supplementation on skeletal growth rate and first-lactation milk production. J. Dairy Sci. 93:2639-2650. https://doi.org/10.3168/jds.2009-3007.

Moco, S., S. Collino, S. Rezzi, and F.-P. J. Martin. 2013. Metabolomics perspectives in pediatric research. Pediatr. Res. 73:570-576. https://doi.org/10.1038/pr.2013.1.

Patti, G. J., O. Yanes, and G. Siuzdak. 2012. Metabolomics: the apogee of the omics trilogy. Nat. Rev. Mol. Cell Biol. 13:263-269. https://doi.org/10.1038/nrm3314.

Ramsay, R. R. 2000. The carnitine acyltransferases: modulators of acyl-CoA-dependent reactions. Biochem. Soc. Trans. 28:182-186. https://doi.org/10.1042/bst0280182.

Ramsay, S. L., W. M. Stoeggl, K. M. Weinberger, A. Graber, and W. Guggenbichler. 2007. Apparatus and Method for Analyzing a Metabolite Profile. Patent No. EP 1875401 A2. Biocrates Life Sciences AG, Innsbruck, Austria, assignee.

Rico, J. E., V. V. R. Bandaru, J. M. Dorskind, N. J. Haughey, and J. W. McFadden. 2015. Plasma ceramides are elevated in overweight Holstein dairy cows experiencing greater lipolysis and insulin resistance during the transition from late pregnancy to early lactation. J. Dairy Sci. 98:7757-7770. https://doi.org/10.3168/jds.2015 -9519 .

Schooneman, M. G., F. M. Vaz, S. M. Houten, and M. R. Soeters. 2013. Acylcarnitines: Reflecting or inflicting insulin resistance? Diabetes 62:1-8. https://doi.org/10.2337/db12-0466.

Shamay, A., D. Werner, U. Moallem, H. Barash, and I. Bruckental. 2005. Effect of nursing management and skeletal size at weaning on puberty, skeletal growth rate, and milk production during first lactation of dairy heifers. J. Dairy Sci. 88:1460-1469. https://doi .org/10.3168/jds.S0022-0302(05)72814-9.

Siskos, A. P., P. Jain, W. Römisch-Margl, M. Bennett, D. Achaintre, Y. Asad, L. Marney, L. Richardson, A. Koulman, J. L. Griffin, F. Raynaud, A. Scalbert, J. Adamski, C. Prehn, and H. C. Keun. 2017. Interlaboratory reproducibility of a targeted metabolomics platform for analysis of human serum and plasma. Anal. Chem. 89:656-665. https://doi.org/10.1021/acs.analchem.6b02930.

Soberon, F., E. Raffrenato, R. W. Everett, and M. E. Van Amburgh. 2012. Preweaning milk replacer intake and effects on long-term productivity of dairy calves. J. Dairy Sci. 95:783-793. https://doi .org/10.3168/jds.2011-4391.

Taylor, E. M., A. D. Jones, and T. M. Henagan. 2014. A review of mitochondrial-derived fatty acids in epigenetic regulation of obesity and type 2 diabetes. J. Nutrit. Health Food Sci. 2:1-4. https://doi .org/10.15226/jnhfs.2014.00127.

Wilson, M. L., S. R. McCoski, A. J. Geiger, R. M. Akers, S. E. Johnson, and A. D. Ealy. 2017. The influence of postnatal nutrition on reproductive tract and endometrial gland development in dairy calves. J. Dairy Sci. 100:3243-3256. https://doi.org/10.3168/jds .2016-11880.

Xia, J., I. V. Sinelnikov, B. Han, and D. S. Wishart. 2015. MetaboAnalyst 3.0-Making metabolomics more meaningful. Nucleic Acids Res. 43:W251.7. https://doi.org/10.1093/nar/gkv380.

Yambayamba, E. S., M. A. Price, and G. R. Foxcroft. 1996. Hormonal status, metabolic changes, and resting metabolic rate in beef heifers undergoing compensatory growth. J. Anim. Sci. 74:57. https:// doi.org/10.2527/1996.74157x.

Zanton, G. I., and A. J. Heinrichs. 2005. Meta-analysis to assess effect of prepubertal average daily gain of Holstein heifers on firstlactation production. J. Dairy Sci. 88:3860-3867. https://doi.org/ 10.3168/jds.S0022-0302(05)73071-X. 